\title{
Military Requirements Development based on Synthetic Microanalytic Approach
}

\author{
Xiaolei Zheng \\ The Logistics College, Beijing, P.R. China \\ Ji Ren \\ The Equipment Demonstration Center, Beijing, P.R. China
}

\begin{abstract}
This paper analyzes the synthetic microanalytic approach (SMA) and its principles in the systematics field, applies those principles to military requirements development, and proposes a method of military requirements development based on synthetic microanalytic approach (MRDSMA). The development methods were analyzed using knowledge from the graph theory, the MRDSMA graph was defined, and the MRDSMA development model was constructed.

KEYWORD: synthetic microanalytic approach; requirements development; requirements model; requirements indicators
\end{abstract}

\section{INTRODUCTION}

The weaponry system is a large complicated system; it is an integration of a variety of weapons and equipment entities. The constituent elements are complex, the application environment is capricious, and the number of participants is huge. The close connection within capabilities, such as battlefield awareness, information domination, logistical support and precision striking can affect the overall function and external capacity of weapons, and it is difficult to reflect the requirements of complex weaponry systems using the traditional requirements analysis and description method. The existing weaponry requirements development methods mainly study the requirements development of a single type of weaponry, without systematic consideration of the role and contribution of a single weapon in the weaponry system. Therefore, the description of relationship within elements of the weaponry system and the description of capability of the weaponry system cannot reflect the joint warfighting capability requirements. The problem regarding the method of integration of simplicity and complexity as well as military and technology urgently needs to be addressed.

\section{INTEGRATED MICROANALYSIS OVERVIEW}

SMA contains two types of interpretation about the system behavior: the macroscopic interpretation expressed in the terms of system theory (rep2), and the microscopic interpretation that associates system and component theory (rep1, rep2, mm, mc, ST-CT), which combines the "top-down" and "bottom-up" point of view; the microscopic interpretation essentially simultaneously applies CT and ST. The concept of component in system expression is interpreted as the microscopic mechanism (mm); the concept of system in component expression is interpreted as the macro constraint (mc).

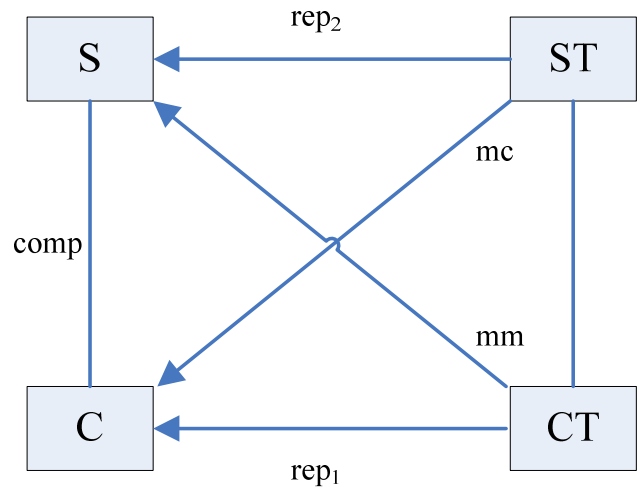

Figure 1 Synthetic Microanalytic Approach Diagram

\section{DEVELOPMENT MECHANISM OF MRDSMA}

\subsection{Characteristics and guidelines of MRDSMA}

Military requirements development is a process from macro to micro, and again from micro to macro. It has the following characteristics:

The macroscopic interpretation of a problem can guide the microscopic analysis; 
The microscopic analysis result of a problem is a more specific description of the macroscopic issue, and meanwhile it cannot go beyond the scope of the macro issue.

MRDSMA must follow the following four criteria:

The objective of macroscopic description of a problem is to obtain its microscopic description. Therefore, it is necessary to start from the macro interpretation of the problem.

The objective of analysis of a problem on the micro-level is to make the macro description clearer. The analysis should proceed to the point where the macroscopic problem can be broken down into a series of specific micro problems, and continue until the accurate answer is found.

In military requirements development, the macroscopicproblemis often required to be broken down to a number of layers. When analyzing a problem of any level, it is necessary to verify the relationship between the macro and micro levels.

In consideration of the complexity of the problem, the number of decomposition layers should be as small as possible. In the decomposition, if the problem can be solved in this layer, it will not be decomposed.

\subsection{MRDSMA principles}

The MRDSMA diagram uses a graphical way to explain the principles of MRDSMA. Suppose a macroscopic problem s cannot be solved directly; then it can be decomposed into two micro problems ${ }^{c_{1}}$ and ${ }^{c_{2}}$. The solution to $c_{1}$ is $f\left(c_{1}\right)$, and the solution to $c_{2}$ is $f$ $\left(c_{2}\right)$. Through the synthesis of $f\left(c_{1}\right)$ and $f\left(c_{2}\right)$, eventually the solution $f(s)$ to the macro problems can be obtained. This solution can be interpreted as an SMA principle, as shown in Figure 2.

The following are some explanations of SMA diagram $G(s, f(s))$ :

The upper level is the macro-level of the problem, and the lower level is the micro-level;

Node $\mathrm{s}$ is the military mission model space $\mathrm{O}$ in the military requirements development core element model. The front section of the triangular pyramid ( $s$, $c_{1}, c_{2}$ ) composites the domain of the problem Q;

Node $f(s)$ is the military requirements system $\mathrm{R}$ in the military requirements development core element model. The back section of the triangular pyramid ( $f$ (s), $\left.f\left(c_{1}\right), f\left(c_{2}\right)\right)$ composites the solution domain $\mathrm{P}$;

The dotted line indicates that there is a conceptual link between the two nodes connected.

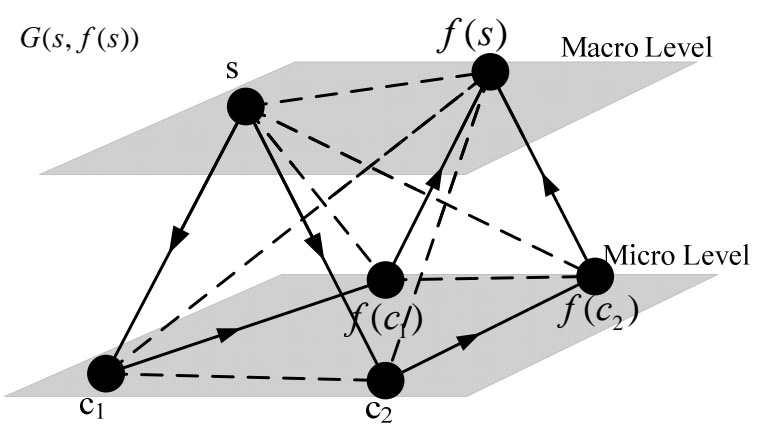

Figure 2 MRDSMA Diagram

For the MRDSMA diagram, the following characteristics can be provided:

Accessibility: The necessary and sufficient condition for node $a$ to reach node $b$ is that there is a directed solid line pointing to node $b$ from node $a$. If node $a$ can reach node $b$, it is expressed as $a \rightarrow b$. Every node is defined to be able to reach itself, namely, $a \rightarrow b$ is always true. If node $a$ cannot reach node $b$, it is expressed as $\neg(a \rightarrow b)$.

Relativity: The necessary and sufficient condition for node $a$ to relate to node $b$ is that there exists a directed solid or dotted line connecting node $a$ and node $b$. If node $a$ andnode $b$ are related, it is expressed as $a \sim b$. Relativity is reversible, namely, $a \sim b \Leftrightarrow a \sim b$. If node $a$ is not related to node $b$, it is expressed as $\neg(a \sim b)$.

Transitivity: If node $a$ can reach node $b$, and node $b$ can reach node $c$, then node $a$ can reach node $c$, namely, $(a \rightarrow b) \wedge(b \rightarrow c) \Rightarrow a \rightarrow c$.

Effectiveness: The necessary and sufficient condition for the SMA diagram $G(s, f(s))$ to be effective is that starting from the initial node $S$, all nodes in the problem domain can be reached. If the diagram $G(s, f$ $(s))$ is effective, it is expressed as $\diamond G(s, f(s))$. The effectiveness can be expressed as:

$\diamond G(s, f(s)) \Leftrightarrow \forall a \in Q . s \rightarrow a$.

On the contrary, the necessary and sufficient condition for $G(s, f(s))$ to be ineffective is that there exists one or more nodes that cannot be reached when starting from the initial node s. If the diagram $G(s, f$ $(s)$ ) is ineffective, it is expressed as $\neg \diamond G(s, f(s))$, and this relationship can be expressed as: $\neg \diamond G(s, f(s)) \Leftrightarrow \exists a \in Q . \neg(s \rightarrow a)$.

Interconnectivity: The necessary and sufficient condition for the SMA diagram $G(s, f(s))$ to be interconnected is that starting from the initial node, all nodes in the diagram can be reached. If the diagram $G(s, f(s))$ is interconnected, it is expressed as ${ }^{\circ} G(s, f(s))$. This can be expressed as: ${ }^{\circ} G(s, f(s)) \Leftrightarrow \forall a \in G(s, f(s)) . s \rightarrow a$.

Interconnectivity can also be described as the necessary and sufficient condition for the SMA diagram $G(s, f(s))$ to be interconnected is that starting from any node in the diagram, the ending node can be reached. This can be expressed as: ${ }^{\circ} G(s, f(s)) \Leftrightarrow \forall a \in G(s, f(s)) . a \rightarrow f(s)$. 
Effectiveness is a necessary condition of interconnectivity, and interconnectivity is a sufficient condition for effectiveness.

\subsection{MRDSMA development process}

The development process MRDSMA is the process of establishing a diagram $G(s, f(s))$ with interconnectivity. This process includes proposing overall objectives, objective decomposition, sub-objective traversal, requirements acquirement, and requirements gathering as shown in Figure 3.

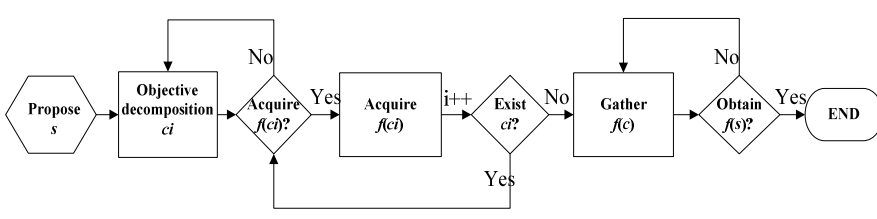

Figure 3 Process of MRDSMA

The specific steps in this process are as follows:

Step One: Put forward the objective. Users from the military force propose the overall objective according to warfighting requirements. The overall objective is usually a rough description of the problem by the warfighting units, which corresponds to $S$ in the $G(s, f(s))$ diagram.

In the field of requirements development, in accordance with the synthesis prior to analysis, the overall objective can be defined as follows:

$S=(U, A, V, \varphi)$

Where $\mathrm{U}$ is non-empty finite set of objects, $U=\left\{X_{1}, X_{2} \ldots X_{\mathrm{n}}\right\}$; A is a non-empty finite set of attributes, $A=\left\{a_{1}, a_{2} \ldots a_{\mathrm{n}}\right\} ; V=\cup_{a \in A} V_{a}$ is a range set of attributes, where $V_{a}$ is the range of attributes; $f$ is the overall objective function of requirements,

$$
\varphi: U \times A \longrightarrow V, \varphi(x, a) \in V_{a} \text {. }
$$

Step Two: After proposing the problem s, the duty of the military requirements development personnel is to establish a clear and accurate system of military requirements, corresponding to $f(s)$ in the $G(s, f(s))$ diagram. There is a conceptual link between $s$ and $f$ $(s)$, which can be understood as $f(s)$ is a function ofs. In general, there is no technology directly froms to $f$ $(s)$, and this relationship betweens and $f(s)$ is indicated by a dotted line.

Step Three: Requirements development personnel should find ways to build the descriptive relationship between sand $f(s)$, and somehow deduce $f$ (s)froms. In the MRDSMA development method, the solution to the problem is to break down $S$ into more specific problems ${ }^{C}$. The purpose is to eventually obtain $(s)$, and $f(s)$ has a conceptual constraint on c. This is also the meaning of the dotted line between $f(s)$ and $c_{1}, c_{2}$ in the $G(s, f(s))$ diagram.

In the field of requirements development analysis, the indiscernibility relation can be used to describe this step; that is, for a subset $B \subseteq A$, the indiscernibility relation of $\mathrm{B}$ on $\mathrm{U}$ is defined as follows:

$\operatorname{IND}(B)=\left\{\left(x, x^{\prime}\right) \in U \times U \mid \forall a \in B, a(x)=a\left(x^{\prime}\right)\right\}$

The indiscernibility relation is an equivalence relation, which divides $U$ into a finite number of sets called equivalence classes. $U / I N D(B)$ is used to indicate all the equivalence classes of $U$.

Assume $S$ is divided into $n$ subsets $c_{1}, c_{2} \ldots c_{\mathrm{n}}$, so there is $\cup_{i=1}^{n} c_{i}=s$ and $\forall i, j . c_{i} \cap c_{j}=\phi$. The dotted lines in $G(s, f(s))$ between $c_{1}$ and $c_{2}$ represents the equivalence relationship between $c_{1}$ and $c_{2}$.

For convenience, assume $n=2$, so there is $s=c_{1} \cup$ $c_{2}, c_{1} \cap c_{2}=\phi$.

Step Four: For the two microscopic problemc1 and c2 of the macro problem $\mathrm{S}$, the requirements development personnel must provide the solutions $\mathrm{f}$ (c1) andf (c2) to the problems accordingly. If by some technical means, f (ci) can be obtained fromci, then the decomposition of cican be stopped; otherwise, ci should be decomposed again.

Step Five: At this step, the tree structure of the descriptive problem has been obtained. Each node in the tree has obtained solutions using some type of technical expression, and now the goal is the bottom-up synthesis. Select a leaf node problem that belongs to a parent node, and integrate the solution into the upper-level solution. Use the sub-nodes $c_{1}$ and $c_{2}$ of $S$ as an example: first, because $c_{1}$ and $c_{2}$ belong to the same parent node $S$, the solutions of $c_{1}$ and $c_{2}\left(f\left(c_{1}\right)\right.$ and $f$ $\left.\left(c_{2}\right)\right)$ are chosen to be synthesized, with the goal of obtaining the solution $f(s)$ of the parent node $s$. Therefore, the synthesis process is constrained bys. This conceptual link between s and $f\left(c_{1}\right), f\left(c_{2}\right)$ is described in the $G(s, f(s))$ diagram as a dotted line connecting $s$ and $f\left(c_{1}\right), f\left(c_{2}\right)$.

Repeat step five, until the solution $f(s)$ of the overall problem $S$ on the top level is obtained. Now the $G(s, f(s))$ diagram is an interconnected diagram. This is the end of the requirements development, and $f(s)$ is the military requirements system.

\section{MRDSMA DEVELOPMENT MODEL}

\subsection{Development Model}

The purpose of the MRDSMA development model is to provide appropriate solutions for the different issues arising from the process of requirements development of the weaponry system. The development model is shown in Figure 4. It consists of three layers, the requirements system layer, the warfighting requirements layer, and the functional requirements layer. Between the layers is the relationship between microscopic analysis and macroscopic synthesis. 


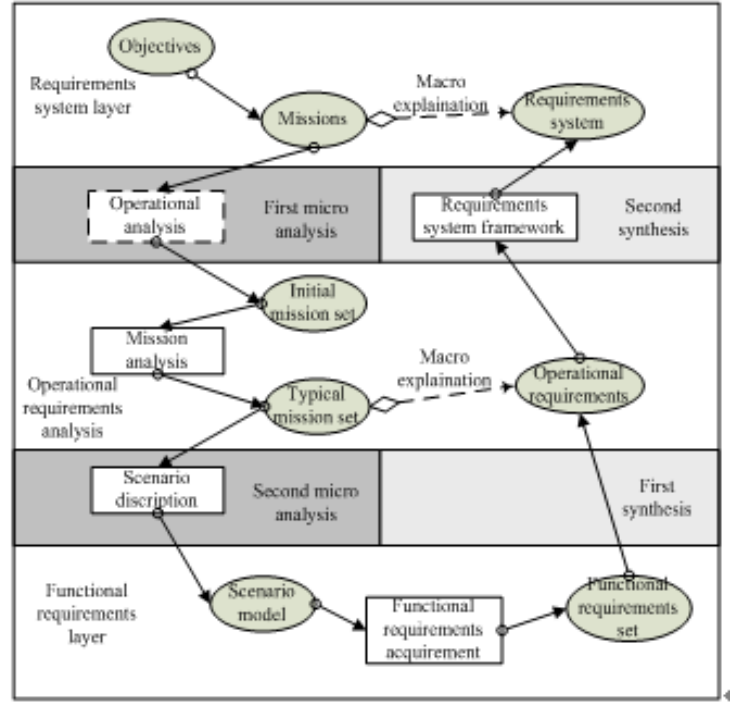

Figure 4 MRDSMA development model

\subsection{Model Structure}

Requirements system layer: In the MRDSMA development model, the requirements system layer is used as a big complicated system. The missions and tasks of the target system are the starting point of the whole requirements development process, and the corresponding requirements system is the end of development.

Warfighting requirements layer: The warfighting requirements layer is a microscopic description of the requirements system layer, but it is also a synthesis of the functional requirements layer. The warfighting requirements layer describes the warfighting missions that the system needs to achieve, as well as the corresponding warfighting requirements.

Functional Requirements layer: In MRDSMA, the functional requirements layer is the lowest layer of microscopic analysis. In this layer, the development personnel must define what specific functions the system should have. The functional requirements layer is a microscopic description of the requirements system layer.

First microscopic analysis: In accordance with the integrated microscopic thinking, when the problem is too complex and it is difficult to obtain its solution at the macro level, it should be decomposed. The decomposition method for the mission is warfighting analysis, in the process of which the tasks that the system needs to perform are specified, and an initial set of tasks are established.

Second microscopic analysis: For the system development personnel, a detailed description of requirements is desired, which provides a description of functions of the system. Studying typical tasks and establishing the scenario of the system upon completion of typical tasks is a way to obtain functional requirements.
First synthesis: The first synthesis is a conceptual synthesis, and the corresponding relationship between the warfighting requirements and the functional requirements must be clearly obtained.

Second synthesis: This is the process of consolidating the warfighting requirements of the system and obtaining the military requirements system. The sorting is based on the relationship between the different warfighting requirements and the corresponding functional requirements.

\subsection{Model Development Process}

Warfighting analysis: This is the process from initial analysis of system tasks to the acquisition of the initial task set of the system, indicated by the dotted lines in the diagram.

Task analysis: This is the process of removing redundant tasks from the initial task set, and merging similar tasks. The product of this step is a set of typical tasks.

Scenario modeling: This is the process of establishing scenario models for the system to perform tasks. The product of this step is a system scenario.

Functional requirements acquisition: This is the process of obtaining the system functional requirements. Its product is the functional requirements set.

Requirements system establishment: This is the process of structuralizing the obtained requirements, and generating the military requirements system.

\section{CONCLUSIONS}

This paper introduced a synthetic microanalytic approach for issues in the requirements development process of the weaponry system, such as the overcomplicated requirement description, the lack of a visual requirements development model and the lack of a clear and concise requirements index system, and proposed a method of military requirements development based on synthetic micro analytic approach (MRDSMA). The characteristics, guidelines, and principles of the military requirements development have been described, the synthetic microanalytic process of military requirements have been defined, and the military requirements development based on synthetic microanalytic approach has been established. The military requirements development was divided into multiple layers from macro to micro, and a different requirement acquisition method was used on each layer. This ensures that the requirements development result can reflect the tasks of the weaponry system as a whole, and maintain the feasibility of requirements development and the accuracy of the development results when it comes to details. 


\section{REFERENCES}

CJCSI $3170.01 C$. Joint capabilities integration and development system.Washington D.C.: Joint Chiefs of Staff, 2003

Department of Defense Architecture Framework Working Group, version 2.0. Washington D.C.:Department of Defense 2009

HU Xiao-feng, YANG Jing-yu, WU Lin, SI Guangya.Weapon System of Systems (SoS) Capabilities Requirement Developmentand Exploratory Simulation Analysis Experimentation.Journal of System Simulation, 2008,20(12): 3065-3068

Jamshidi M.Systems of systems engineering-principles and applications.UK:CRC Press,2009

Jamshidi M.Systems of systems engineering-invovations for the 21st century.USA:Wildy,2009

Keating C, Rogers R, UnalR , et al. System of systems engineering. Engineering Management Joural, 2003, 15(3): 36-35

Luiz Marco Cysneiros,and Julio Cesar. NonfunctionalRequirements:From Elicitation to Concepetual Models. IEEE Trans. 2014, 30(25): 328-340

Macaulay L. Requirement Engineering. London: Springer, 1996. 26-29

Maier M W .Architecting principles for system of system. Systems Engineering,2008,1(6): 267-284

Youssef R,KimB,et al.Toward an integrated executable architecture and based analysis for counter terrorism and homeland security.Ottawa:Defense R\&D Canada Otttawa future forces synthetic environment,2006

ZHAO Qing-song, HUANG Wei, LU Yan-jing, CHEN Yingwu.A Study on Task and Capability of Weapon System of System Based on Concept Lattice. Journal of System Simulation, 2009,21(12): 3782-3784 